\title{
Sucesso a partir de investimento em metodologias de gestão de projetos
}

\author{
Leandro Alves Patah ${ }^{\text {a* }}$, Marly Monteiro de Carvalho ${ }^{\text {b }}$ \\ a*leandro.patah@uol.com.br, UNINOVE, Brasil \\ ${ }^{\mathrm{b}}$ marlymc@usp.br, POL1/USP, Brasil
}

\begin{abstract}
Resumo
0 objetivo deste trabalho é investigar o relacionamento entre a adoção de metodologias de gestão de projetos e o sucesso em projetos. A abordagem metodológica envolveu um levantamento de campo longitudinal, conduzido em três países, Argentina, Brasil e Chile, por um período de 3 anos em uma única organização com amplo acesso às informações. Foram obtidos dados de 1.387 projetos. Os resultados demonstram influência positiva e significativa das variáveis capacitação e número de PMPs (Project Management Profissionals) no sucesso dos projetos e influência positiva do uso de metodologias de projetos no desempenho em prazo, indicando benefícios para a organização estudada.
\end{abstract}

Palavras-chave

Administração de projetos. Indicadores de desempenho. Modelos de maturidade.

\section{Introdução}

0 gerenciamento de projetos, como teoria, encontra-se bem desenvolvido e é aceito nos dias de hoje no mercado como uma competência necessária para as organizações. Vários métodos e técnicas têm sido desenvolvidos cobrindo todos os aspectos de um projeto, desde sua concepção até a entrega final dos produtos produzidos por ele. Apesar disso, a gestão de projetos permanece um grande desafio, uma vez que uma grande quantidade de projetos excede seu orçamento, atrasa ou falha em cumprir seus objetivos, como evidenciado por diversas pesquisas (Dai \& Wells, 2004; Standish Group International, 2009; White \& Fortune, 2002).

0 The Standish Group (Standish Group International, 2009), baseando-se em uma pesquisa com 280.000 projetos de tecnologia de informação, mostra que apenas 32\% dos projetos podem ser considerados um sucesso. Próximo da metade dos projetos pesquisados, isto é, 44\%, apresenta problemas de prazos ou custos e $24 \%$ já não podem mais ser recuperados e foram considerados projetos com falhas. Segundo a mesma pesquisa, os projetos que gastam mais do que o valor previsto estouram seu orçamento, em média, em 45\%. Quanto a completar o projeto no prazo, os dados da pesquisa também não são nada animadores: em média, o cronograma é ampliado em 63\% de seu prazo original, sendo que somente $67 \%$ das características requeridas e funcionalidades são normalmente entregues aos clientes dos projetos.

Nos últimos anos, muitas empresas têm gasto quantidades significativas de recursos em projetos. 0 PMI (Project Management Institute, 2009) apresenta um número de US\$12 trilhões, um quinto do valor do PIB mundial, como o valor a ser investido em projetos em cada um dos anos da atual década.

Apesar disso, não se encontra uma grande quantidade de pesquisas a respeito do retorno do investimento em métodos de gerenciamento de projetos. Com isso, mesmo com o alto grau de investimento em projetos e o crescente número de gerentes de projetos qualificados, algumas questões sobre os resultados e benefícios desses investimentos surgem. Em geral, trabalhos de revisão da teoria de projetos apresentam, entre outras, as seguintes perguntas (lka, 2009; Söderlund, 2004): 
- Como provar que gastar dinheiro em métodos e capacitação em gerenciamento de projetos tem valor e qual a função ou valor adicionado pela gestão de projetos?

- Como tornar os altos executivos comprometidos com os métodos de gerenciamento de projetos sem mostrar os resultados financeiros gerados em decorrência dos investimentos realizados?

- 0 que determina o sucesso ou a falha de um projeto?

As poucas evidências quantitativas existentes apresentam um cenário nada animador. Os projetos falham e isso não vai mudar, a menos que as empresas comecem a medir onde os projetos falham e por quê (Buchanan, 2008).

Apesar do volume de literatura existente na área, ainda há poucas evidências empíricas que relacionem implementação de gerenciamento de projetos aos resultados obtidos. Por outro lado, os executivos também buscam evidências de que seus investimentos estão funcionando efetivamente e produzindo o valor esperado de lucros ao final dos projetos. Em pesquisas recentes sobre a medição do valor e do sucesso do gerenciamento de projetos, a questão de se quantificar seu valor ainda não se encontra satisfatoriamente respondida (lka, 2009; Thomas \& Mullaly, 2008).

Com o objetivo de contribuir para a avaliação dos benefícios do gerenciamento de projetos nas organizações, este trabalho busca avaliar a relação entre os investimentos realizados e os resultados dos projetos. Os investimentos na implementação de gerenciamento de projetos envolvem o desenvolvimento e uso de ferramentas e métodos, treinamento de gerentes de projetos e suporte administrativo e organizacional. Como proxy de retorno obtido será utilizado o desempenho operacional alcançado pelos projetos. A abordagem metodológica envolve um estudo longitudinal, com estratégia híbrida, qualitativa e quantitativa.

Este artigo está estruturado em cinco seções. A seção 2 traz a síntese do quadro teórico, seguida da seção 3, que apresenta a abordagem metodológica. Os resultados e as discussões são apresentados na seção 4. Por fim, a seção 5 traz as conclusões e recomendações para trabalhos futuros.

\section{Revisão de literatura}

\subsection{Métodos de gerenciamento de projetos}

Projetos podem ser definidos como empreendimentos "esquecíveis" conduzidos dentro de organizações, que surgem em meio à rotina, sendo compostos por indivíduos que dificilmente irão trabalhar juntos novamente. Uma estratégia para codificar as capacidades da organização em gestão de projetos deve ser definida no nível da empresa para repetir abordagens de sucesso em próximos projetos. Utilizando-se um processo bem estruturado e implementado, essas capacidades podem ser armazenadas e transferidas ao longo do tempo, do espaço e do contexto. Adicionalmente, por meio da criação de uma memória externa aos indivíduos, esse tipo de codificação de conhecimento pode tornar as organizações menos vulneráveis à perda do conhecimento tácito armazenado nas pessoas (lbert, 2004).

Uma sistemática de projetos pode ser composta por métodos, pacotes de ferramentas e modelos de projetos. Desse modo, a gestão de projetos pode ser vista como a aplicação sequencial de processos estruturados, repetidos e contínuos que, quando utilizados por uma organização de forma gradual e segura para seus negócios, permite dar passos rumo à institucionalização de práticas padronizadas.

A sistemática precisa auxiliar a equipe no planejamento e entrega dos projetos, considerando o ciclo inteiro de vida de forma consistente e eficiente, sempre orientada para os negócios e para a satisfação dos clientes (Silveira, 2008). E, para isso, há algumas características das sistemáticas de gerenciamento de projetos das organizações destacadas pelo autor, como estar em sintonia com a 1509000 ou com outras normas de institutos oficiais de gerenciamento de projetos. Deve também ter um conjunto compreensivo e flexível de processos, ferramentas e técnicas que deem suporte às atividades, auditadas periodicamente por um escritório de gestão de projetos (Project Management Office - PMO). Ressalta-se ainda a necessidade de documentação, instrumentos de medição e controle de projetos durante o ciclo de vida e comunicação dos resultados aos stakeholders.

Há vários conjuntos de modelos de métodos de gerenciamento de projetos disponíveis para utilização por profissionais e organizações para melhor gerenciar seus projetos. Os padrões atualmente mais difundidos são disponibilizados por institutos e associações dedicados ao estudo de projetos, conforme apresentado na Tabela 1.

De modo geral, o que se observa é que o PMBoK é um conjunto de práticas de gestão de projetos genérico e bastante abrangente que objetiva atender às necessidades dos mais diversos tipos de projetos (Project Management Institute, 2008). Por isso mesmo deve ser adaptado quando da aplicação de seus conceitos por parte das organizações. Durante a adaptação, é grande a chance de a organização se perder no grau de profundidade com que deve tratar os 42 processos presentes no documento, 
frente às suas necessidades específicas. Além disso, se a organização também conduz programas, deve buscar os conceitos adicionais e específicos na norma de programa publicada pelo PMI.

A norma europeia de gerenciamento de projetos, publicada pelo IPMA, o ICB, se configura como uma excelente fonte de referência para aqueles que buscam uma opção de métodos em gerenciamento de projetos mais voltada para os aspectos humanos da gestão (International Project Management Association, 2006). Estruturado por competências que o projeto necessita desenvolver, seu conteúdo encontra-se dividido em três partes: competências contextuais, comportamentais e técnicas.

Outros dois guias de referência de associações ainda pouco difundidos em organizações brasileiras são o australiano AIPM (Australian Institute of Project Management, 2008) e o inglês APM Body of Knowledge (Association for Project Management, 2006). 0 guia do instituto australiano de projetos configura-se como uma boa fonte de referência para os aspectos humanos do gerenciamento de projetos. 0 instituto inglês de gerenciamento de projetos apresenta um dos mais completos conjuntos de métodos pesquisados neste trabalho. Além dos aspectos relacionados à gestão técnica de projetos, esse documento apresenta conteúdos que tratam os conceitos do valor do gerenciamento de projetos, dos modelos e sistemáticas de implementação de escritórios de projetos e dos aspectos estratégicos da gestão de projetos.

Mais voltado ao mercado de tecnologia de informação, o PRINCE2 apresenta-se estruturado por etapas de um projeto e por atividades a serem conduzidas pela sua equipe de gestão, por isso é um pouco mais voltado à aplicação prática do que os demais (Office of Government Commerce, 1996).

0 ENAA, publicado pelo instituto japonês de gerenciamento de projetos, dedica-se a analisar com mais profundidade os aspectos técnicos e contratuais de grandes projetos de engenharia tendo, portanto, uma aplicação mais restrita a determinados segmentos de mercado (Engineering Advancement Association of Japan, 1992).

A Tabela 2 apresenta de uma forma resumida os conjuntos de métodos em gerenciamento de projetos, suas principais características e diferenças dos demais.

\subsection{Desempenho em projetos}

A efetividade do processo de gerenciamento de projetos irá determinar se os projetos desempenham um forte papel em fornecer uma fonte de vantagem competitiva para uma organização (Barber, 2004).

Mesmo as organizações que aparentemente apresentam sucesso nos seus projetos, possuem uma inconsistência latente e uma falta de habilidade para identificar o problema e a maneira de reduzi-lo (Buchanan, 2008). Um primeiro passo para resolver o problema é padronizar o que significa uma performance satisfatória e que o inimigo natural de uma performance de excelência consistente é a disparidade. Para Buchanan (2008), em geral as métricas se dividem em duas categorias: medidas preditivas que ajudam a prever ou identificar tendências funcionando como um sistema de alerta inicial e medidas corretivas que auxiliam a estabelecer padrões para melhorar a performance em projetos futuros.

Diversos trabalhos têm sido conduzidos, nesta última década, buscando analisar como o sucesso em projetos pode ser medido (Belout \& Gauvreau, 2004; Besner \& Hobbs, 2006; Bizan, 2003; Dvir et al., 2003; Gray, 2001; Kendra \& Taplin, 2004; Lipovetsky et al., 2005; Raz et al., 2002; Repiso et al., 2007).

0 sucesso em projetos é usualmente definido como cumprir os objetivos de tempo, custo e qualidade e satisfazer os stakeholders do projeto.

Tabela 1. Principais padrões propostos por associações de gerenciamento de projetos.

\begin{tabular}{|c|c|c|c|}
\hline Instituto & Conjunto de métodos & País de origem & Foco da metodologia \\
\hline $\begin{array}{l}\text { Project Management Institute } \\
\text { (PMI) }\end{array}$ & $\begin{array}{l}\text { Project Management Body of } \\
\text { Knowledge (PMBoK) }\end{array}$ & EUA & Gestão geral de projetos \\
\hline $\begin{array}{c}\text { International Project } \\
\text { Management Association (IPMA) }\end{array}$ & ICB - IPMA Competence Baseline & União Europeia & Gestão geral de projetos \\
\hline $\begin{array}{l}\text { Australian Institute of Project } \\
\text { Management (AIPM) }\end{array}$ & $\begin{array}{c}\text { AIPM - Professional Competency } \\
\text { Standards for Project } \\
\text { Management }\end{array}$ & Austrália & Gestão geral de projetos \\
\hline $\begin{array}{l}\text { Association for Project } \\
\text { Management (APM) }\end{array}$ & APM Body of Knowledge & Reino Unido & Gestão geral de projetos \\
\hline $\begin{array}{l}\text { Office of Government Commerce } \\
\qquad(\mathrm{OGC})\end{array}$ & $\begin{array}{l}\text { Projects in Controlled } \\
\text { Environments (PRINCE2) }\end{array}$ & Reino Unido & $\begin{array}{c}\text { Gestão de projetos de sistemas de } \\
\text { informação }\end{array}$ \\
\hline $\begin{array}{c}\text { Japan Project Management } \\
\text { Forum (JPMF) }\end{array}$ & $\begin{array}{l}\text { ENAA Model Form-International } \\
\text { Contract for Process Plant } \\
\text { Construction }\end{array}$ & Japão & Gestão de projetos de construções \\
\hline
\end{tabular}


Tabela 2. Os conjuntos de métodos em gerenciamento de projetos e suas principais características.

\begin{tabular}{|c|c|c|}
\hline Conjunto de métodos & Características & Diferenças dos demais \\
\hline $\begin{array}{l}\text { PMBoK - Project Management Body } \\
\text { of Knowledge }\end{array}$ & $\begin{array}{l}\text { Conjunto de métodos desenvolvidos para diversos } \\
\text { tipos de projetos, sendo, portanto, bastante genérico. } \\
\text { Estruturado por áreas de conhecimento de um projeto. }\end{array}$ & $\begin{array}{l}\text { É complementado por dois conjuntos de } \\
\text { métodos adicionais: Programa e Portfólio. }\end{array}$ \\
\hline 1CB - IPMA Competence Baseline & $\begin{array}{c}\text { Estruturado por competências que o projeto necessita } \\
\text { desenvolver, divididas em: contextuais, comportamentais } \\
\text { e técnicas. }\end{array}$ & $\begin{array}{l}\text { Juntamente com a norma australiana, } \\
\text { apresenta um grau de profundidade } \\
\text { bem maior que os demais métodos nos } \\
\text { aspectos humanos da figura do gerente } \\
\text { do projeto. }\end{array}$ \\
\hline $\begin{array}{l}\text { AIPM Professional Competency } \\
\text { Standards for Project Management }\end{array}$ & $\begin{array}{l}\text { Esse documento, publicado pelo instituto australiano de } \\
\text { projetos, é bastante similar em sua estrutura ao PMBoK, } \\
\text { dividido por áreas de conhecimento. }\end{array}$ & $\begin{array}{l}\text { Também possui um enfoque mais } \\
\text { profundo nas habilidades humanas. }\end{array}$ \\
\hline APM Body of Knowledge & $\begin{array}{l}\text { Um dos mais completos conjuntos de métodos, esse } \\
\text { documento apresenta conteúdos relacionados a projetos, } \\
\text { valor, escritório de projetos e aspectos estratégicos da } \\
\text { gestão de projetos. }\end{array}$ & $\begin{array}{l}\text { É o mais abrangente dos conjuntos de } \\
\text { métodos. }\end{array}$ \\
\hline $\begin{array}{c}\text { PRINCE2 - Projects in Controlled } \\
\text { Environments }\end{array}$ & $\begin{array}{l}\text { Conjunto de métodos estruturados por etapas de um } \\
\text { projeto e nas atividades a serem conduzidas pela sua } \\
\text { equipe de gestão. }\end{array}$ & $\begin{array}{l}\text { Conjunto de métodos mais voltado para } \\
\text { projetos de tecnologia de informação. }\end{array}$ \\
\hline $\begin{array}{l}\text { ENAA Model Form-International } \\
\text { Contract for Process Plant } \\
\text { Construction }\end{array}$ & $\begin{array}{l}0 \text { documento tem um enfoque muito grande nos } \\
\text { aspectos contratuais de um projeto. }\end{array}$ & $\begin{array}{l}0 \text { foco desse conjunto de métodos são } \\
\text { projetos de construção em engenharia. }\end{array}$ \\
\hline
\end{tabular}

Apesar disso, as pesquisas conduzidas ao longo dos últimos anos e desde os anos 1980 que vêm investigando as dimensões do sucesso em projetos levaram à reescritura da fórmula acima com a concordância geral de que o sucesso em projetos é multidimensional e que diferentes pessoas medem o sucesso de projetos de diferentes maneiras em épocas diferentes (Barber, 2004; Bryde, 2003; 1ka, 2009; Jugdev \& Muller, 2005).

Com o objetivo de medir o sucesso em projetos é possível cria critérios e métricas, como proposto por ling (2004), que faz uma divisão refinando o sucesso em projetos entre atingir sucesso no produto do projeto através do atendimento de padrões de qualidade e atingir o sucesso no processo por meio do cumprimento de objetivos de tempo e orçamento. Para isso, são utilizadas quatro métricas para avaliar a performance dos projetos: custo, tempo, qualidade e atendimento à satisfação do cliente.

Já Larson \& Gobeli (1989) apresentam alguns fatores que podem afetar o sucesso de projetos, como: estrutura do projeto, competência do gerente do projeto e tamanho do projeto, utilizando os mesmos indicadores propostos por ling (2004).

Métricas trazem consistência e formalidade à gestão de projetos. Com métricas, decisões importantes de projeto podem ser tomadas com base em informações. Em essência, métricas trazem objetividade às ferramentas para o monitoramento do progresso de projetos e ajudam melhorar a organização por permitir uniformidade, acuracidade e repetibilidade (Rad \& Levin, 2006). Com este intuito, Rad \& Levin
(2006) propõem métricas para a empresa, para as pessoas e para o próprio projeto.

Outra maneira de avaliar o sucesso é desdobrá-lo em dois critérios diferentes. Um, o próprio sucesso, que, segundo Cooke-Davies (2002), não pode ser medido até que o projeto seja finalizado, e outro, a performance de projetos, que pode ser medida durante a execução. Segundo o autor, nenhum sistema de métricas em projetos pode ser considerado completo sem um dos pacotes de medidas (performance e sucesso) e deve-se buscar um método de ligá-los, como um meio de avaliar a acuracidade, com a qual a performance dos projetos prediz o sucesso da organização.

Thamhain (2004), em um estudo conduzido entre 2000 e 2003 com 76 times de projetos de 27 empresas, busca, por sua vez, associar o ambiente de equipes de projetos com a sua performance. Segundo o autor, as principais variáveis relacionadas aos times de projetos que influenciam o sucesso são o ambiente do time e a performance da equipe.

Os benefícios gerados para o cliente (Dvir et al., 1998), a adaptabilidade e a habilidade do projeto de cooperar com outras áreas da organização (Katz \& Allen, 1985) e o atendimento de padrões de qualidade e segurança (White \& Fortune, 2002) também devem ser inclusos em sistemas de medição de desempenho de projetos.

Critérios financeiros vêm sendo utilizados para medir a performance em projetos já há algum tempo. Os critérios podem incluir retorno econômico, análise custo/benefício (Archer \& Ghasemzadeh, 1999), contribuição da melhoria de medidas financeiras tais 
como lucros, market share e valor de novos projetos obtidos (Thomas et al., 2002).

Outra maneira direta de se avaliar os benefícios do gerenciamento de projetos é analisar as margens dos projetos correntes de uma empresa. É possível comparar a margem de um projeto quando a empresa o vende com o valor obtido quando ele é concluído. A diferença pode ser explicada em parte pela metodologia de gerenciamento de projetos (Patah \& Carvalho, 2007).

Para ter um valor real, medidas de performance devem ser cuidadosamente alinhadas com objetivos organizacionais claros, tais como eficiência, produtividade ou previsibilidade. Porém, a simples presença de um sistema de medição, que pode nunca ser perfeito, irá causar melhorias na performance dos projetos. Quando alguém decide que alguma atividade necessita ser medida, então ela se torna importante e as pessoas começam a se preocupar com ela. E esse fato, mesmo que isoladamente, irá por si só melhorar a performance dos projetos (Buchanan, 2008).

A Tabela 3 apresenta o resumo das métricas para medição de performance em projetos pesquisadas em várias fontes para a elaboração deste trabalho.

Pode-se observar que as métricas para medição da performance em projetos mais utilizadas são aquelas relacionadas à obtenção, ao final do projeto, dos valores inicialmente planejados de prazo e custo (Gray, 2001; Katz \& Allen, 1985; Larson \& Gobeli, 1989; ling, 2004; White \& Fortune, 2002), sendo que é consenso a questão financeira envolvida (Archer

Tabela 3. Métricas para medição de performance em projetos.

\begin{tabular}{|c|c|}
\hline Autor & Métricas para medição de performance em projetos \\
\hline Katz \& Allen (1985) & $\begin{array}{l}\text { - Cronograma; } \\
\text { - Orçamento; } \\
\text { - Performance de custo; } \\
\text { - Inovação no projeto; } \\
\text { - Adaptabilidade; } \\
\text { - Habilidade em cooperar com outras áreas da organização. }\end{array}$ \\
\hline Larson \& Gobeli (1989) & $\begin{array}{l}\text { - Controle dos custos; } \\
\text { - Cumprimento de prazos; } \\
\text { - Performance técnica. }\end{array}$ \\
\hline Dvir et al. (1998) & $\begin{array}{l}\text { - Cumprimento das metas definidas; } \\
\text { - Benefícios para o cliente. }\end{array}$ \\
\hline Archer \& Ghasemzadeh (1999) & $\begin{array}{l}\text { - Retorno econômico; } \\
\text { - Análise custo/benefício; } \\
\text { - Riscos; } \\
\text { - Impacto no mercado. }\end{array}$ \\
\hline Gray (2001) & $\begin{array}{l}\text { - Orçamento; } \\
\text { - Cronograma; } \\
\text { - Especificação técnica; } \\
\text { - Opinião dos stakeholders. }\end{array}$ \\
\hline White \& Fortune (2002) & $\begin{array}{l}\text { - Requerimentos de clientes atendidos; } \\
\text { - Completado dentro do cronograma; } \\
\text { - Completado dentro do orçamento; } \\
\text { - Objetivos organizacionais atendidos; } \\
\text { - Negócios fortalecidos; } \\
\text { - Descontinuidades nos negócios minimizadas; } \\
\text { - Padrões de qualidade e segurança atendidos. }\end{array}$ \\
\hline 1bbs \& Reginato (2002) & - Valor medido pelo processo. \\
\hline Thomas et al. (2002) & $\begin{array}{l}\text { - Contribuição da melhoria de medidas financeiras; } \\
\text { - Melhoria do desempenho das equipes de projetos; } \\
\text { - Melhoria da satisfação dos consumidores, dos lucros e do market share; } \\
\text { - Valor de novos projetos obtidos. }\end{array}$ \\
\hline Ling (2004) & $\begin{array}{l}\text { - Custo; } \\
\text { - Tempo; } \\
\text { - Qualidade; } \\
\text { - Atendimento à satisfação do cliente. }\end{array}$ \\
\hline Thamhain (2004) & $\begin{array}{l}\text { - Ambiente do time do projeto; } \\
\text { - Performance do time do projeto. }\end{array}$ \\
\hline Rad \& Levin (2006) & $\begin{array}{l}\text { - Empresa; } \\
\text { - Pessoas; } \\
\text { - Coisas. }\end{array}$ \\
\hline Patah \& Carvalho (2007) & $\begin{array}{l}\text { - Medida da comparação entre o custo de um projeto quando a companhia inicia o mesmo com o } \\
\text { valor obtido quando o projeto é concluído. }\end{array}$ \\
\hline
\end{tabular}


\& Ghasemzadeh, 1999; Patah \& Carvalho, 2007; Thomas et al., 2002), o que já era esperado. Alguns desses autores, porém, incluem outros conceitos como riscos (Archer \& Ghasemzadeh, 1999), satisfação de stakeholders, novos projetos obtidos e desempenho da equipe (Thomas et al., 2002). E outros fornecem uma visão um pouco distinta ao incluir o valor como uma função dos processos que as organizações geram (lbbs \& Reginato, 2002).

\subsection{Impacto das metodologias e práticas de gestão de projetos no sucesso}

Compreender o impacto do gerenciamento de projetos no desempenho da organização ainda é uma lacuna de pesquisa, segundo Aubry \& Hobbs (2010). Apesar do crescimento das pesquisas nessa área nas últimas décadas, as dimensões de desempenho para a medição do sucesso continuam tratadas no triângulo de ferro (escopo, prazo e custo) (PapkeShields et al., 2010).

Besner \& Hobbs (2012) comparam os padrões dominantes de utilização das práticas, ferramentas e técnicas com o proposto no guia PMBoK. Embora os autores identifiquem certo alinhamento, os autores observam que o guia PMBoK parece exagerar em algumas práticas em detrimento de outras. Por exemplo, os conjuntos de práticas associadas ao grupo de processos de inicialização têm utilização de moderada a extensa, mas recebem tratamento resumido no PMBoK. Em estudo mais recente, Besner \& Hobbs (2013) sugerem que existem imbricações diversas entre maturidade e competência e o sucesso. Em ambos os estudos, os autores reforçam a interveniência da variável tipo de projetos tanto na adoção das práticas quanto em suas consequências nos resultados.

Chou \& Yang (2012) analisam o impacto do guia PMBoK no desempenho do projeto, satisfação do cliente e sucesso do projeto no setor de construção civil em Taiwan, concluindo que é conveniente a adoção das práticas do PMBoK na indústria da construção, em especial na área de gestão de aquisições. $\mathrm{Na}$ mesma linha, McHugh \& Hogan (2011) discutem os porques da adoção de uma metodologia reconhecida internacionalmente em gestão de projetos em dois perfis de organização; naquelas em que existe uma metodologia de gerenciamento de projetos e nas que não têm uma metodologia de gerenciamento de projetos. Os autores argumentam que as razões dessa adoção são os benefícios do uso de uma metodologia internacionalmente reconhecida, que incluem: a garantia de que a organização está usando o que é considerado a melhor prática, a demanda de clientes externos para que uma metodologia reconhecida seja usada, assistência no recrutamento externo e disponibilidade de fornecedores de treinamento e suporte na metodologia. Já Sanchez-Losado (2012) contrapõem o PMBoK e o pensamento enxuto (Lean Thinking) no contexto de projetos de construção, argumentando que os projetos com certo nível de incerteza não podem ser geridos pautando-se apenas no PMBoK, mas devem incorporar aspectos do pensamento, compatibilizando os métodos.

No contexto nacional, Berssaneti et al. (2012) encontraram impacto dos modelos de referência e maturidade no gerenciamento de projetos de Tecnologia da Informação (Tl), houve correlação positiva entre o grau de maturidade organizacional em gerenciamento de projetos e a satisfação das demandas dos stakeholders, bem como entre a adoção do PMBoK e o atendimento dos requisitos e satisfação das demandas dos stakeholders. Moraes \& Kruglianskas (2012) corroboram essa visão, pois concluem que as organizações com maior nível de maturidade em gestão de projetos criam condições para que o desempenho de seus projetos fique menos dependente da ação individual dos gerentes.

Alves et al. (2012) também investigaram o tema, mas sob a perspectiva das melhores práticas, concluindo que há impacto positivo da adoção de melhores práticas na implantação de escritórios de gerenciamento de projetos com o sucesso. Outros estudos nacionais também focam em práticas, mas em áreas específicas como risco (Rabechini Jr. \& Carvalho, 2012) e comunicação (Carvalho \&t Mirandola, 2007), e também verificam impacto significativo e positivo em algumas dimensões de sucesso.

\section{Metodologia e hipóteses}

\subsection{Modelo conceitual}

Para a medição da performance dos projetos neste trabalho, os critérios de medição de desempenho são divididos em operacionais e estratégicos. Os critérios de desempenho operacional são aqueles relacionados a atingir sucesso no produto do projeto, através do atendimento de padrões de qualidade, e atingir sucesso no processo de gestão do projeto, através do cumprimento de objetivos de tempo e orçamento. Os critérios de desempenho estratégico são decorrentes de definições externas aos projetos, relacionadas com o ambiente organizacional em que o projeto está inserido, em que se busca atingir o sucesso esperado pelo cliente e outros stakeholders do projeto.

Vários fatores podem levar a um alto desempenho em projetos, como visto anteriormente. Neste estudo, enfatiza-se o uso de métodos de gerenciamento de projetos (Kessler \& Winkellhofer, 2002; Standish 
Group International, 2009; White \& Fortune, 2002) e o treinamento e a capacitação de equipes de projetos (Cooke-Davies, 2002; Dai \& Wells, 2004). Dessa forma, foram selecionados os seguintes aspectos para análise neste trabalho:

- Possuir um sistema de gerenciamento de projetos formal com métodos bem definidos;

- Capacitação, designação de responsabilidades e treinamento do gestor do projeto e dos membros da equipe.

Dos indicadores de medição do desempenho discutidos na seção anterior, são considerados neste trabalho:

- Cumprimento do orçamento planejado;

- Cumprimento do prazo planejado;

- Performance financeira do projeto relacionada às análises e decisões de investimento realizadas antes da aprovação do projeto.

\subsection{Hipóteses da pesquisa}

\subsubsection{Uso de métodos de gerenciamento de projetos}

Vários autores enumeram os benefícios do uso de métodos de gerenciamento de projetos (lbert, 2004; Kerzner, 2001; Kessler \& Winkellhofer, 2002; Standish Group International, 2009; White \& Fortune, 2002). Kerzner (2001) apresenta uma lista de benefícios, entre eles: a melhoria no cumprimento das atividades em relação aos planos e o atendimento aos objetivos do projeto. lbert (2004), quando comenta as características dos projetos, apresenta o conceito de que o uso de métodos padronizados de gestão de projetos pode fazer com que a empresa repita abordagens de sucesso em próximos projetos. Com o objetivo de medir quantitativamente o impacto da adoção de métodos de gerenciamento de projetos na sua performance, a primeira hipótese deste trabalho é formulada da seguinte maneira:

- H1: 0 grau de utilização de métodos de gerenciamento de projetos não influencia o resultado operacional dos projetos.

Derivam dessa primeira hipótese as seguintes hipóteses:

- H1a: O grau de utilização de métodos de gerenciamento de projetos não influencia no cumprimento do prazo dos projetos.

- H1b: O grau de utilização de métodos de gerenciamento de projetos não influencia no cumprimento do custo.
- H1c: O grau de utilização de métodos de gerenciamento de projetos não influencia na performance financeira dos projetos.

\subsubsection{Esforço em treinamento e capacitação em gerenciamento de projetos}

Archibald (2003) apresenta o gerente do projeto como sendo ele próprio um dos fatores críticos de sucesso do projeto. Adicionalmente, Cooke-Davies (2002) inclui em sua relação de fatores críticos de sucesso para um projeto a capacitação adequada em todos os níveis da organização nos conceitos de gestão de projeto. Outros autores, também, incluem questões de treinamento e capacitação como fatores chave de sucesso em gestão de projetos (Dai \& Wells, 2004). Para avaliar se o investimento em treinamento e capacitação em gerenciamento de projetos melhora o seu desempenho foram estabelecidas as hipóteses $\mathrm{H} 2$ e $\mathrm{H} 3$.

- H2: 0 investimento em treinamento e capacitação em gerenciamento de projetos não influencia o resultado operacional dos projetos.

Derivam dessa segunda hipótese as seguintes hipóteses:

- H2a: 0 investimento em treinamento e capacitação em gerenciamento de projetos não influencia no cumprimento do prazo dos projetos.

- H2b: 0 investimento em treinamento e capacitação em gerenciamento de projetos não influencia no cumprimento do custo.

- H2c: 0 investimento em treinamento e capacitação em gerenciamento de projetos não influencia na performance financeira dos projetos.

\subsubsection{Número de gerentes de projetos certificados como PMP}

A terceira hipótese deste trabalho é apresentada da seguinte forma:

- H3: 0 número de gerentes de projetos certificados como PMP na divisão de condução do projeto não influencia o resultado operacional dos projetos.

Derivam dessa terceira hipótese as seguintes hipóteses:

- H3a: 0 número de gerentes de projetos certificados como PMP não influencia no cumprimento do prazo dos projetos.

- H3b: 0 número de gerentes de projetos certificados como PMP não influencia no cumprimento do custo.

- H3c: 0 número de gerentes de projetos certificados como PMP não influencia na performance financeira dos projetos. 
Todas as hipóteses nulas contêm sempre uma igualdade sobre a qual deve-se obter evidências para rejeitá-la. 0 nível de significância $(\alpha)$, fixado neste estudo em 5\%, representa a probabilidade (ou risco) de incorrer-se no erro de rejeitar $\mathrm{HO}$ quando essa hipótese é de fato verdadeira.

\subsection{Seleção da amostra de análise}

Conforme sugerido por Eisenhardt \& Graebner (2007), foi utilizada a amostragem teórica (theoretical sampling). Como o foco da pesquisa são organizações em que a atividade de projetos é estratégica, e, por conseguinte, investem grandes montas em métodos e treinamentos em gestão de projetos, esse foi o recorte teórico utilizado para definição da amostra. Em trabalhos que estudaram a performance em projetos, pouca evidência foi encontrada em organizações com pouco histórico de gestão de projetos (Bryde, 2003; Silveira, 2008).

Além disso, para a análise longitudinal, tinha-se como requisito o acesso aos dados de desempenho do projeto em uma base temporal significativa para permitir a comparação do desempenho dos projetos antes, durante e após a implantação dos métodos, em uma abordagem similar à utilizada por Hendricks \& Singhal (2001). Dados para tratar as questões de valores em projetos não estão facilmente disponíveis na maioria das organizações (Thomas \& Mullaly, 2008). Ao optar-se por uma única organização, com amplo acesso às informações, buscou-se reduzir sensivelmente esse risco da indisponibilidade de informações normalmente tratadas como confidenciais.

Por fim, necessitava-se de uma base comum para comparação do avanço da implantação, do uso dos métodos e da maturidade em projetos. Dada a grande quantidade de diferentes métodos disponíveis para a gestão de projetos, conforme apresentado na seção 2, caso a escolha recaísse sobre várias organizações seria necessário equalizar esses diversos métodos adotados ou adaptados pelas empresas para obter-se uma base comparável de variáveis independentes. Além disso, em decorrência das inúmeras possibilidades de medição do sucesso em projetos, também discutidas na seção 2 , seria necessário gerar uma base comparável para as variáveis dependentes, conforme apresentado por Pinto (2002).

A organização estudada é uma empresa multinacional com diversas divisões atuando em diferentes mercados, em que foi possivel obter dados de uma grande quantidade de projetos de um longo período de análise. Essa empresa tem $60 \%$ de suas vendas brutas provenientes de projetos. A empresa produz e instala uma grande variedade de equipamentos, na sua maioria entregues aos clientes por meio de projetos específicos, adaptados às necessidades de cada um. A taxa de inovação dos produtos é extremamente alta e os produtos atualmente vendidos foram desenvolvidos no máximo 3 anos atrás. Basicamente, a empresa vende soluções customizadas para seus clientes, com baixo volume de unidades produzidas e uma grande variedade.

0 programa de implementação de gestão de projetos nas unidades de negócio da empresa na região do Mercosul data de 2003, com o início dos cursos de certificação, estruturação do PMO e difusão da metodologia da matriz. Desde então, a sistemática de compartilhamento das melhores práticas, a criação de padrões em gerenciamento de projetos, a busca de um número suficiente de gerentes de projeto qualificados e a uniformização da cultura de gerenciamento de projetos na empresa foram feitas visando atingir-se a rentabilidade sustentada nas operações.

A empresa tem diversas unidades de negócio e para o dimensionamento da amostra de projetos analisados foi adotada a regra prática proposta por Hair et al. (1998): quando se trata da análise de dependência ou interdependência entre variáveis, deve-se obter, pelo menos, 20 amostras para cada variável estudada, o que totalizou 120 amostras de projetos a serem obtidas durante a etapa de coleta de dados.

Neste trabalho decidiu-se utilizar a amostragem não-probabilística para o levantamento dos dados dos projetos, uma vez que são esses a unidade de análise adotada. Essa escolha se justifica pelo fato de que, com esta pesquisa, não se busca estimar, através da amostra, os parâmetros da população e, sim, identificar relações entre variáveis, onde a amostragem não probabilística se mostra eficiente, uma vez que o problema a ser tratado é menos sensível à não aleatoriedade da amostra. Mesmo que fosse planejada uma amostra probabilística para selecionar os respondentes, o envio dos formulários aos respondentes não garantiria que o conjunto de dados constituiria uma amostra probabilística, uma vez que a decisão de responder ou não cabe exclusivamente aos respondentes, que decidiriam se fariam ou não parte da amostra. $\mathrm{Ou}$ seja, mesmo que para este trabalho se buscasse obter dados de projetos através de formulários enviados aos gerentes de projetos, não se teria a garantia de que a amostragem seria probabilística.

Com o objetivo de capturar os resultados dos projetos e o nível de aplicação de metodologias nos mesmos foi criado um instrumento preenchido pelos gestores da organização e auditado pelo setor de auditoria da empresa, o qual permitiu identificar a performance de projetos por meio de indicadores de desempenho de prazos e custos e explorar o grau 
de aplicação e utilização de métodos de gestão aos projetos. Esse instrumento é apresentado no Anexo 1.

\subsection{Operacionalização das variáveis da pesquisa}

0 sucesso dos projetos foi medido com o objetivo de avaliar o benefício gerado por meio do uso de métodos e capacitação em gerenciamento de projetos das equipes que os gerenciam e, também, mais especificamente, dos gerentes dos projetos. Procura-se, com isso, medir a influência que o esforço e investimento em gerenciamento de projetos geram na obtenção de um maior retorno financeiro pelas empresas. Espera-se que essa correlação permita medir a melhoria no desempenho de uma empresa pelo uso do gerenciamento de projetos. Para todos os projetos foram obtidas as informações relacionadas no Anexo 2.

Os investimentos na implementação de gerenciamento de projetos envolvem o desenvolvimento e uso de ferramentas e métodos, treinamento de gerentes de projetos e suporte administrativo e organizacional. Como proxy de retorno foi utilizado o desempenho operacional obtido pelos projetos, avaliado através dos critérios de resposta.

0 grau de implementação dos métodos de gestão de projetos é avaliado nas diversas unidades de negócio da empresa em uma escala de 0 a 100\% por um departamento de auditoria central.

0 presente trabalho define variáveis independentes e dependentes segundo o conceito de Marconi \& Lakatos (2003).
Como a variável independente $(X)$ é aquela que influencia, determina ou afeta outra variável, de acordo com as hipóteses, foram escolhidas as variáveis independentes de uso de métodos de gerenciamento de projetos e de esforço em treinamento e capacitação em gerenciamento de projetos.

Uma vez que a variável dependente $(Y)$ consiste naqueles valores (fenômenos ou fatores) a serem explicados ou descobertos, em virtude de serem influenciados, determinados ou afetados pela variável independente, a escolha do cumprimento de custos, prazos e a performance financeira do projeto baseou-se nos principais indicadores de medição de desempenho selecionados da teoria, conforme discutido anteriormente.

Neste trabalho, as variáveis independentes são medidas contra as variáveis dependentes, de acordo com as hipóteses 1,2 e 3.

A Tabela 4 apresenta as variáveis selecionadas para este trabalho e suas respectivas formas de medição e grandeza.

A Tabela 5 apresenta as hipóteses e os resultados esperados das relações entre as variáveis independentes e dependentes.

Para a análise dos dados obtidos utilizou-se um software específico de análise estatística que atendia plenamente as necessidades do trabalho, o Statistical Package for the Social Sciences (SPSS).

\section{Discussões e análise dos resultados}

Foram obtidos dados de 2.851 projetos conduzidos no período de janeiro de 2005 a junho de 2008 .

Tabela 4. Variáveis e formas de medição.

\begin{tabular}{|c|c|c|}
\hline Variável & Forma de medição & Grandeza \\
\hline $\begin{array}{l}\text { X1 - Uso de métodos de gerenciamento de } \\
\text { projetos }\end{array}$ & $\begin{array}{c}\text { Grau de implementação de gerenciamento de projetos medido } \\
\text { em uma escala de } 0 \text { a } 100 \% \text {, onde cada um dos } 12 \text { módulos } \\
\text { do programa da empresa tem sua implementação avaliada } \\
\text { pelo setor de auditoria da empresa. } 0 \text { valor resultante final } \\
\text { é calculado por meio da média simples entre os } 12 \text { valores } \\
\text { obtidos. }\end{array}$ & $\%$ \\
\hline $\begin{array}{l}\text { X2 - Esforço em treinamento e capacitação em } \\
\text { gerenciamento de projetos }\end{array}$ & $\begin{array}{l}\text { Valor financeiro do investimento em treinamento e capacitação } \\
\text { em gerenciamento de projetos e quantidade de gerentes de } \\
\text { projetos certificados na área de gestão do projeto. Foi utilizada } \\
\text { a certificação PMP como variável de medição por ser essa o } \\
\text { foco da capacitação em gestão de projetos da empresa. }\end{array}$ & $\mathrm{R} \$$ e valor numérico \\
\hline Y1 - Cumprimento de orçamento & $\begin{array}{c}\text { Variação relativa do aumento de orçamento, medida pela } \\
\text { diferença em valor monetário do orçamento original previsto } \\
\text { pelo projeto em relação ao orçamento real final do projeto, } \\
\text { dividida pelo orçamento original. }\end{array}$ & $\%$ \\
\hline Y2 - Cumprimento de prazo & $\begin{array}{l}\text { Variação relativa do aumento de prazo do projeto, medida } \\
\text { através da diferença entre o prazo original planejado em dias e } \\
\text { o prazo final do projeto, dividida pelo prazo original. }\end{array}$ & $\%$ \\
\hline Y3 - Performance financeira & $\begin{array}{l}\text { Variação relativa da margem do projeto, medida através da } \\
\text { diferença entre a margem final do projeto e a margem prevista } \\
\text { originalmente, dividida pela margem original. }\end{array}$ & $\%$ \\
\hline
\end{tabular}


Tabela 5. Hipóteses de pesquisa e influências a serem verificadas.

\begin{tabular}{|c|c|c|c|}
\hline Hipótese & Variável independente (X) & Variável dependente (Y) & Resultado esperado \\
\hline $\mathrm{H} 1$ & $\begin{array}{l}\text { X1 - Uso de métodos de gerenciamento de } \\
\text { projetos }\end{array}$ & $\begin{array}{c}\text { Y1 - Cumprimento de orçamento } \\
\text { Y2 - Cumprimento de prazo } \\
\text { Y3 - Performance financeira }\end{array}$ & Influencia \\
\hline $\mathrm{H} 2$ & $\begin{array}{c}\text { X2 - Esforço em treinamento e capacitação em } \\
\text { GP (investimento financeiro) }\end{array}$ & $\begin{array}{c}\text { Y1 - Cumprimento de orçamento } \\
\text { Y2 - Cumprimento de prazo } \\
\text { Y3 - Performance financeira }\end{array}$ & Influencia \\
\hline $\mathrm{H} 3$ & $\begin{array}{c}\text { X2 - Esforço em treinamento e capacitação em } \\
\text { GP (número de PMPs) }\end{array}$ & $\begin{array}{c}\text { Y1 - Cumprimento de orçamento } \\
\text { Y2 - Cumprimento de prazo } \\
\text { Y3 - Performance financeira }\end{array}$ & Influencia \\
\hline
\end{tabular}

Porém, vários desses projetos não apresentavam informações completas como custos finais de execução, a categorização atribuída e outros. Após uma análise detalhada, chegou-se a 1.387 projetos com dados completos, ou 48,65\% do total, possíveis de serem analisados, para se tentar comprovar as hipóteses estabelecidas na seção 3. Esses projetos foram conduzidos entre julho de 2006 e junho de 2008. Para todos os projetos foram obtidas as informações relacionadas no Anexo 2, coletadas através do instrumento apresentado no Anexo 1.

Em relação ao segmento de atuação do projeto, a área com mais projetos obtidos é a área de energia, com 713 projetos ( $51,41 \%$ do total), seguida pela área de medicina, com 218 projetos. Quanto ao país de realização, a maior parte dos projetos foi conduzida no Brasil, com 823 projetos (59,34\% do total), seguido da Argentina, com 294 projetos $(21,20 \%)$ e do Chile, com 270 projetos $(19,47 \%)$.

\subsection{Análise descritiva dos resultados}

\subsubsection{Variáveis independentes}

A variável independente Uso de Métodos de Gerenciamento de Projetos (X1) está concentrada entre $70,83 \%$ e $83,33 \%$ e apresenta uma baixa dispersão relativa (coeficiente de variação $=4 \%$ ), conforme Tabela 6.

De acordo com essa variável, os projetos têm média de 77,18\% de grau de implantação, mediana de 75,98\%, moda de 75,00\% e desvio padrão de 3,29\%.

A variável independente Esforço em treinamento e capacitação em gerenciamento de projetos (Investimento financeiro) (X2) apresenta valores compreendidos entre $\mathrm{R} \$ 0,00$ e $\mathrm{R} \$ 615.000,00$, com frequência relativa baixa em todo o intervalo e com distinção para os valores extremos (nenhum investimento e R\$ 615.000,00), conforme Tabela 7.

Os projetos apresentam, em média, investimentos de $\mathrm{R} \$ 238.609,00$, com mediana de $\mathrm{R} \$ \mathbf{8 5 . 0 0 0 , 0 0}$ e moda de $R \$ 615.000,00$. A dispersão relativa é de
Tabela 6. Distribuição de frequência da variável independente X1.

\begin{tabular}{ccc}
\hline Grau de implantação & Frequência & $\%$ \\
\hline $70,83 \%$ & 71 & 5,12 \\
$73,81 \%$ & 122 & 8,80 \\
$75,00 \%$ & 294 & 21,20 \\
$75,93 \%$ & 162 & 11,68 \\
$75,98 \%$ & 178 & 12,83 \\
$77,60 \%$ & 150 & 10,81 \\
$80,83 \%$ & 137 & 9,88 \\
$80,95 \%$ & 110 & 7,93 \\
$82,64 \%$ & 87 & 6,27 \\
$83,33 \%$ & 76 & 5,48 \\
Total & 1.387 & 100,00 \\
\hline
\end{tabular}

Tabela 7. Distribuição de frequência da variável independente X2 (Investimento).

\begin{tabular}{ccc}
\hline Investimento financeiro & Frequência & $\%$ \\
\hline $\mathrm{R} \$ 0,00$ & 252 & 18,17 \\
$\mathrm{R} \$ 5.000,00$ & 73 & 5,26 \\
$\mathrm{R} \$ 15.000,00$ & 93 & 6,71 \\
$\mathrm{R} \$ 25.000,00$ & 107 & 7,71 \\
$\mathrm{R} \$ 45.000,00$ & 36 & 2,60 \\
$\mathrm{R} \$ 50.000,00$ & 11 & 0,79 \\
$\mathrm{R} \$ 55.000,00$ & 52 & 3,75 \\
$\mathrm{R} \$ 60.000,00$ & 13 & 0,94 \\
$\mathrm{R} \$ 70.000,00$ & 35 & 2,52 \\
$\mathrm{R} \$ 80.000,00$ & 14 & 1,00 \\
$\mathrm{R} \$ 85.000,00$ & 131 & 9,44 \\
$\mathrm{R} \$ 130.000,00$ & 62 & 4,47 \\
$\mathrm{R} \$ 295.000,00$ & 22 & 1,59 \\
$\mathrm{R} \$ 470.000,00$ & 41 & 2,96 \\
$\mathrm{R} \$ 500.000,00$ & 13 & 0,94 \\
$\mathrm{R} \$ 615.000,00$ & 432 & 31,15 \\
Total & 1.387 & 100,00 \\
\hline
\end{tabular}

113\% (coeficiente de variação), com desvio padrão de $\mathrm{R} \$ 270.344,00$.

A variável independente X2 - Esforço em treinamento e capacitação em gerenciamento de projetos (Número de PMPs) apresenta alta concentração de respostas nos extremos, com limite inferior (valor zero) com 32,66\% dos projetos e limite superior (valor 19) com 32,08\% dos projetos, conforme Tabela 8. 
Os projetos têm, em média, sete funcionários com certificação e mediana de três funcionários com certificação. A dispersão relativa é de 116\% (coeficiente de variação) e desvio padrão de oito.

\subsubsection{Variáveis dependentes}

A variável dependente $\mathrm{Y} 1$ - Cumprimento de orçamento distribuí-se num intervalo entre mínimo de $-100 \%$ (valor final maior do que valor estimado) e 716,6\% (valor final menor do que valor estimado). Adicionalmente, quase metade dos projetos empata o custo final com o planejado e poucos projetos $(12,98 \%)$ têm seu custo final acima do planejado, conforme Tabela 9.

Os projetos têm uma variação média de custo de 9,51\% abaixo do estimado, com mediana e moda igual a zero. A dispersão relativa (dispersão cerca de quase cinco vezes a média) é alta, com coeficiente de variação de 495\% e desvio-padrão de 47,07\%.

A variável dependente Y2 - Cumprimento de prazo distribui-se num intervalo entre $-10 \%$ (projeto atrasado) e $+10 \%$ (projeto adiantado). A maioria dos projetos $(70,23 \%)$ é finalizada dentro da data planejada. Os valores são apresentados na Tabela 10.

Os projetos têm prazo final, em média de 4,66\% abaixo do esperado, mediana e moda de 9,8\% abaixo do inicialmente estimado. A dispersão relativa é alta, com coeficiente de variação de $159 \%$ e desvio padrão de 7,42\%.

A variável dependente $Y 3$ - Performance financeira distribui-se num intervalo entre $-664 \%$ (projetos indicando prejuízo) e 502\% (projetos indicando ganho), conforme Tabela 11.

Para essa variável, a média é de projetos com performance financeira negativa $(-2,68 \%)$, com mediana e moda iguais a zero. Os projetos têm alta variabilidade, com desvio padrão de 84,40\%.

Levando-se em consideração variação de custo, variação de prazo e variação de margem, pode-se dizer que o maior problema na gestão dos projetos aqui analisados se dá em variação de prazo.

\subsection{Análise das hipóteses}

Foi conduzida uma análise de correlação, por meio do coeficiente de Pearson, conforme resultados apresentados na Tabela 12. Verifica-se que as variáveis X2 (lnvestimento financeiro) e X2 (Número de PMPs) estão altamente correlacionadas $(\mathrm{r}=0,975)$. Salvo essa correlação, todas as demais são consideradas muito baixas, conforme escala adotada neste trabalho e apresentada na Tabela 13.

Observa-se, portanto, que apenas as variáveis independentes estão correlacionas.
Tabela 8. Distribuição de frequência da variável independente X2 (Número de PMPs).

\begin{tabular}{ccc}
\hline Número de PMPs & Frequência & $\%$ \\
\hline 0 & 453 & 32,66 \\
1 & 167 & 12,04 \\
2 & 65 & 4,69 \\
3 & 127 & 9,16 \\
6 & 103 & 7,42 \\
9 & 22 & 1,59 \\
10 & 5 & 0,36 \\
19 & 445 & 32,08 \\
Total & 1.387 & 100,00 \\
\hline
\end{tabular}

Tabela 9. Distribuição de frequência da variável dependente Y1.

\begin{tabular}{ccc}
\hline $\begin{array}{c}\text { Indice de variação } \\
\text { relativa de custo }\end{array}$ & Frequência & $\%$ \\
\hline Negativo & 180 & 12,98 \\
Zero & 667 & 48,09 \\
Positivo & 540 & 38,93 \\
Total & $\mathbf{1 . 3 8 7}$ & 100,00 \\
\hline
\end{tabular}

Tabela 10. Distribuição de frequência da variável dependente Y2.

\begin{tabular}{ccc}
\hline $\begin{array}{c}\text { Indice de variação } \\
\text { relativa de prazo }\end{array}$ & Frequência & $\%$ \\
\hline Negativo & 412 & 29,70 \\
Zero & 1 & 0,07 \\
Positivo & 974 & 70,23 \\
Total & 1.387 & 100,00 \\
\hline
\end{tabular}

Tabela11. Distribuição de frequência da variável dependente Y3.

\begin{tabular}{ccc}
\hline Performance financeira & Frequência & $\%$ \\
\hline Negativo & 367 & 26,46 \\
Zero & 505 & 36,41 \\
Positivo & 515 & 37,13 \\
Total & 1.387 & 100,00 \\
\hline
\end{tabular}

No entanto, como observado por Pinto (2002), a questão sobre as formas de avaliação de sucesso sempre foi alvo de polêmica em vários trabalhos, uma vez que sucesso é um fenômeno altamente subjetivo e difícil de ser mensurado objetivamente com uma única variável. Com isso, este trabalho tem, assim como Pinto (2002), três alternativas:

- Considerar a medida de sucesso/insucesso como uma variável métrica composta a partir dos valores atribuídos para cada dimensão de sucesso. Dessa forma seria criado um índice $R$ composto pelas $n$ variáveis que medem o sucesso, ou seja, uma variável dependente e $\mathrm{n}$ variáveis independentes. Assim seria possível aplicar a técnica de análise múltipla de variância. A consequência seria ter de assumir como métrica do trabalho uma variável $\mathrm{R}$ que seria o avaliador de sucesso/insucesso; 
- Idem ao item anterior, porém considerando que o índice R (métrico) fosse dicotômico, por exemplo, classificando insucesso se $R<0,5$ e sucesso se $\mathrm{R}>=0,5$;

- Considerar vários indicadores (métricos ou não métricos) para avaliar a dimensão de sucesso em projeto. Nesse caso, ter-se-iam n variáveis dependentes (métricas) como diferentes medidas de sucesso sendo influenciadas por $m$ variáveis independentes (métricas) como diferentes medidas para a metodologia.

Optou-se, por aprofundar a análise, tratando a variável dependente como sucesso/insucesso do projeto como não métrica e dicotômica.

Analisou-se cada uma das variáveis dependentes separadamente e considerou-se um projeto no prazo aquele que apresentou variação zero ou positiva em relação ao planejado. Similar análise foi empreendida para custo e performance financeira.

Em seguida, considerou-se o projeto bem-sucedido se as três variáveis dependentes tivessem variação zero ou positiva em relação ao planejado, ou seja, o projeto é um sucesso se foi concluído no prazo, dentro do orçamento e com a performance financeira planejada.

Os resultados foram analisados a partir do teste não paramétrico de mediana de Mood (Mood Median Test), que não requer que as populações apresentem distribuição normal, dado que as variáveis não apresentam distribuição normal. A Tabela 14 apresenta a síntese dos resultados.

0 teste de Mood mostrou relacionamento significativo e positivo entre a variável dependente prazo e todas as variáveis independentes para um grau de confiança de 99\%. Dessa forma, há indícios que quanto maior o investimento em capacitação, a existência de PMPs e o grau de implementação das metodologias de gestão de projetos, maior a chance do projeto cumprir seu prazo planejado. Por outro lado, nenhuma das variáveis independentes mostrou impacto significativo com relação às variáveis dependentes custo e performance financeira.

Tabela 12. Análise de correlação entre as variáveis (coeficiente de Pearson).

\begin{tabular}{|c|c|c|c|c|c|c|}
\hline Variável & $\mathrm{X} 1$ & X2 (Investim. Financeiro) & X2 (Número de PMPs) & Y1 & $\mathrm{Y} 2$ & Y3 \\
\hline $\mathrm{X} 1$ & 1 & 0,139 & 0,191 & $-0,043$ & 0,181 & $-0,016$ \\
\hline X2 (lnvestimento financeiro) & 0,139 & 1 & 0,975 & $-0,037$ & 0,195 & 0,054 \\
\hline X2 (Número de PMPs) & 0,191 & 0,975 & 1 & $-0,079$ & 0,26 & 0,047 \\
\hline $\mathrm{Y}_{1}$ & $-0,043$ & $-0,037$ & $-0,079$ & 1 & $-0,231$ & 0,018 \\
\hline $\mathrm{Y} 2$ & 0,181 & 0,195 & 0,26 & $-0,231$ & 1 & 0,09 \\
\hline Y3 & $-0,016$ & 0,054 & 0,047 & 0,018 & 0,09 & 1 \\
\hline
\end{tabular}

Tabela 13. Qualificação de acordo com faixa do índice de correlação.

\begin{tabular}{cc}
\hline Qualificação & Faixa do índice de correlação \\
\hline Muito baixo & $0,00-0,29$ \\
Baixo & $0,30-0,49$ \\
Moderado & $0,50-0,69$ \\
Alta & $0,70-0,89$ \\
Muito alta & $0,90-1,00$ \\
\hline
\end{tabular}

Tabela 14. Análise dos relacionamentos entre variáveis.

\begin{tabular}{|c|c|c|c|}
\hline Variável X & Variável $Y$ & Chi-Square & p-value \\
\hline X1 - Uso de métodos de gerenciamento de projetos & Y1 - Cumprimento de orçamento & 2,83 & 0,093 \\
\hline X1 - Uso de métodos de gerenciamento de projetos & Y2 - Cumprimento de prazo & 21,09 & $0,000^{* * * *}$ \\
\hline X1 - Uso de métodos de gerenciamento de projetos & Y3 - Performance financeira & 1,80 & 0,179 \\
\hline X1 - Uso de métodos de gerenciamento de projetos & Sucesso & 3,06 & 0,080 \\
\hline X2 - Esforço em treinamento e capacitação em GP (investimento financeiro) & Y1 - Cumprimento de orçamento & 1,70 & 0,192 \\
\hline X2 - Esforço em treinamento e capacitação em GP (investimento financeiro) & Y2 - Cumprimento de prazo & 19,86 & $0,000^{* * *}$ \\
\hline X2 - Esforço em treinamento e capacitação em GP (investimento financeiro) & Y3 - Performance financeira & 0,15 & 0,694 \\
\hline X2 - Esforço em treinamento e capacitação em GP (investimento financeiro) & Sucesso & 5,21 & $0,022^{* *}$ \\
\hline X2 - Esforço em treinamento e capacitação em GP (número de PMPs) & Y1 - Cumprimento de orçamento & 2,83 & 0,092 \\
\hline X2 - Esforço em treinamento e capacitação em GP (número de PMPs) & Y2 - Cumprimento de prazo & 21,42 & $0,000^{* * * *}$ \\
\hline X2 - Esforço em treinamento e capacitação em GP (número de PMPs) & Y3 - Performance financeira & 0,12 & 0,724 \\
\hline X2 - Esforço em treinamento e capacitação em GP (número de PMPs) & Sucesso & 4,94 & $0,026^{* *}$ \\
\hline
\end{tabular}

\footnotetext{
**Significativo para 95\% de confiança; ***Significativo para 99\% de confiança.
} 
A análise dos projetos considerados sucesso ou fracasso revelou relacionamento positivo e significante com relação às variáveis independentes investimento em capacitação e número de PMPs para um grau de confiança de 95\%. Não obstante, não há relacionamento significativo para a variável independente grau de implementação.

\section{Conclusões}

Este trabalho contribui para sanar uma lacuna na literatura no sentido de se evidenciar o benefício dos investimentos e do uso de métodos de gerenciamento de projetos no sucesso dos projetos, apontada por diversos autores (lka, 2009; Repiso et al., 2007; Thomas \& Mullaly, 2008).

Se, por um lado, o trabalho reúne evidências de que há benefícios com relação a prazo, por outro não foi possivel constatar os benefícios para questões financeiras, quer com relação ao custo, quer com relação à margem.

Para os projetos de sucesso na concepção adotada nesta pesquisa, ou seja, projetos concluídos no prazo, no valor orçado e respeitando as margens planejadas, também foi possível identificar correlação positiva e significante tanto do uso de métodos quanto dos investimentos em capacitação e PMPs.

Este trabalho traz como implicação para a prática gerencial evidências dos benefícios de investimentos em tempo e dinheiro no treinamento e capacitação de equipes de projetos para uma organização multinacional de grande porte. Já o uso de métodos de gerenciamento de projetos não pôde ser totalmente comprovado, havendo apenas indícios de aprimoramento em prazo. Essa constatação alerta para o fato de que provavelmente os métodos estejam sendo mais eficazes com relação à gestão dos prazos do que com relação a questões financeiras. Talvez esse problema resida na métrica utilizada neste trabalho para o uso de métodos, gerando falha de precisão nessa medida, conforme apresentado na Tabela 6 . Sugere-se que esses aspectos sejam explorados em uma agenda futura de estudos. Caso pesquisas adicionais mostrem que a métrica é adequada, outra possível explicação para esse fato seria que o investimento em metodologia de projetos pode trazer benefícios próprios, mesmo no caso do não uso dos métodos ao final. Em tese isso significaria que, com maior capacitação, gestores negociam melhor no decorrer do projeto. E mesmo sem a adoção efetiva dos métodos (práticas formais ou metodologias), isso seria suficiente para resultados de desempenho melhores. A principal limitação desta pesquisa foi o uso de uma única organização como fonte de informação de projetos, embora extraída de 10 unidades de negócio de setores industriais distintos, o que faz com que os resultados não possam ser generalizados. No entanto, essa opção trouxe benefícios que dificilmente seriam obtidos caso se optasse por conduzir análises com diversas organizações. 0 primeiro deles foi a disponibilidade de informações, obtidas diretamente das bases de dados da organização. Foi possível obter uma amostra grande de projetos (1.387), coletada por um extenso período de tempo, com dados detalhados, inclusive a performance financeira, que em geral é tratada como confidencial em grande parte das organizações, Além disso, a variável grau de utilização dos métodos de gestão, para sua confiabilidade, precisava ser feita em uma base sistemática e longitudinal. Dessa forma utilizaram-se dados auditados da empresa analisada.

\section{Referências}

Alves, R. 0., Costa, H. G., Quelhas, 0. L. G., Silva, L. E., \& Pimentel, L. B. (2012). Melhores práticas em implantação de escritório de gerenciamento de projeto: desenvolvimento de referenciais de sucesso. Produção, 23(3), 582-594. http://dx.doi.org/10.1590/ S0103-65132012005000094

Archer, N. P., \& Ghasemzadeh, F. (1999). An integrated framework for project portfolio selection. International Journal of Project Management, 17(4), 207-216. http:// dx.doi.org/10.1016/S0263-7863(98)00032-5

Archibald, R. D. (2003). Managing High-Technology Programs and Projects. New York: John Wiley.

Association for Project Management - APM. (2006). APM Body of Knowledge. Buckinghamshire: APM.

Aubry, M., \& Hobbs, B. (2010). A Fresh Look at the Contribution of Project Management to Organizational Performance. Project Management Journal, 42(1), 3-16. http://dx.doi.org/10.1002/pmj.20213

Australian Institute of Project Management - AIPM. (2008) AIPM Professional Competency Standards for Project Management. Sidney: AIPM.

Barber, E. (2004). Benchmarking the management of projects: a review of current thinking. International Journal of Project Management, 22, 301-307. http:// dx.doi.org/10.1016/j.jproman.2003.08.001

Belout, A., \& Gauvreau, C. (2004). Factors influencing project success: The impact of human resource management. International Journal of Project Management, 22, 1-11. http://dx.doi.org/10.1016/S0263-7863(03)00003-6

Berssaneti, F. T., Carvalho, M. M., \& Muscat, A. R. N. (2012). Impacto dos modelos de referência e maturidade no gerenciamento de projetos: estudo exploratório em projetos de tecnologia da informação. Produção, 22(3), 405-420. http://dx.doi.org/10.1590/ S0103-65132012005000027

Besner, C., \& Hobbs, B. (2013). Contextualized Project Management Practice: A Cluster Analysis of Practices and Best Practices. Project Management Journal, 44(1), 1734. http://dx.doi.org/10.1002/pmj.21291

Besner, C., \& Hobbs, B. (2012). An empirical identification of project management toolsets and a comparison among 
project types. Project Management Journal, 43(5), 2446. http://dx.doi.org/10.1002/pmj.21292

Besner, C., \& Hobbs, B. (2006). The perceived value and potential contribution of project management practices to project success. Project Management Journal, 37(3), 37-48.

Bizan, 0. (2003). The determinants of success of R\&D projects: Evidence from american-israeli research alliances. Research Policy, 32, 1619-1640. http://dx.doi. org/10.1016/S0048-7333(03)00044-1

Bryde, D. J. (2003). Modelling project management performance. International Journal of Quality \& Reliability Management, 20(2), 229-254. http://dx.doi. org/10.1108/02656710310456635

Buchanan, J. (2008). Measuring up. PM Network. Project Management Institute.

Carvalho, M. M., \& Mirandola, D. (2007). A comunicação em projetos de Tl: uma análise comparativa das equipes de sistemas e de negócios. Produção, 17(2), 330-342. http://dx.doi.org/10.1590/S0103-65132007000200009

Chou, J. S., \& Yang, J. G. (2012). Project Management Knowledge and Effects on Construction Project Outcomes: An Empirical Study. Project Management Journal, 43(5), 47-67. http://dx.doi.org/10.1002/ pmj. 21293

Cooke-Davies, T. J. (2002). The "real" success factors on projects. International Journal of Project Management, 20, 185-190. http://dx.doi.org/10.1016/ S0263-7863(01)00067-9

Dai, C. X., \& Wells, W. G. (2004). An exploration of project management office features and their relationship to project performance. International Journal of Project Management, 22, 523-532. http://dx.doi.org/10.1016/j. ijproman.2004.04.001

Dvir, D., Raz, T., \& Shenhar, A. (2003). An empirical analysis of the relationship between project planning and project success. International Journal of Project Management, 21, 89-95. http://dx.doi.org/10.1016/ S0263-7863(02)00012-1

Dvir, D., Lipovetsky, S., Shenhar, A., \& Tishler, A. (1998). In search of project classification: A non-universal approach to project success factors. Research Policy, 27, 915-935. http://dx.doi.org/10.1016/S0048-7333(98)00085-7

Eisenhardt, K. M., \& Graebner, M. E. (2007). Theory building from cases: opportunities and challenges. Academy of Management Journal, 50(1), 25-32. http://dx.doi. org/10.5465/AMJ.2007.24160888

Engineering Advancement Association of Japan - ENAA. (1992). Model Form-International Contract for process plant construction. Tokyo: ENAA.

Gray, R. (2001). Organizational climate and project success. International Journal of Project Management, 19, 103109. http://dx.doi.org/10.1016/S0263-7863(99)00060-5

Hair, J. F., Anderson, R. E., Tatham, R. L., \& Black, W. C. (1998). Multivariate data analysis. New Jersey: Prentice Hall.

Hendricks, K., \& Singhal, V. R. (2001). The long-run stock price performance of firms with effective TQM programs. Management Science, 47(3), 359-368. http://dx.doi. org/10.1287/mnsc.47.3.359.9773 lbbs, W., \& Reginato, J. (2002). Quantifying the value of project management. Project Management Institute Inc., Pennsylvania: Newton Square.

lbert, 0. (2004). Projects and firms as discordant complements: Organisational learning in the Munich software ecology. Research Policy, 33, 1529-1546. http://dx.doi. org/10.1016/j.respol.2004.08.010

lka, L. A. (2009). Project success as a topic in project management journals. Project Management Journal, Four Campus Boulevard: Project Management Institute, 40(4), 06-19.

International Project Management Association - IPMA. (2006). ICB - IPMA Competency Baseline. Nijkerk: IPMA.

Jugdev, K., \& Muller, R. (2005). A retrospective look at our evolving understanding of project success. Project Management Journal, 36(4), 19-31.

Katz, R., \& Allen, T. J. (1985). Project performance and the locus of influence in the R\&D matrix. Academy of Management Journal, 28. http://dx.doi. org/10.2307/256062

Kendra, K., \& Taplin, L. (2004). Project success: A cultural framework. Project Management Journal, 35(1), 30-45.

Kerzner, H. (2001). Project management - A systems approach to planning, scheduling, and controlling. New York: John Wiley \& Sons. PMid:11176335.

Kessler, H., \& Winkellhofer, G. (2002). Projekt-management: Leitfaden zur steuerung und fuhrung von projekten. Heidelberg: Springer. http://dx.doi.org/10.1007/978-3642-97891-3

Larson, E., \& Gobeli, D. (1989). Significance of project management structure on development success. IEEE Transactions on Engineering Management, 36(2), 119125. http://dx.doi.org/10.1109/17.18828

Ling, F. Y. Y. (2004). How project managers can better control the performance of design-build projects. International Journal of Project Management, 22, 477-488. http:// dx.doi.org/10.1016/j.ijproman.2003.09.003

Lipovetsky, S., Tishler, A., Dvir, D., \& Shenhar, A. (2005). The relative importance of project success dimensions. $R \& D$ Management, 27(2), 97-106. http://dx.doi. org/10.1111/1467-9310.00047

Marconi, M., \& Lakatos, E. (2003). Fundamentos de metodologia científica. São Paulo: Atlas.

McHugh, 0., \& Hogan, M. (2011). Investigating the rationale for adopting an internationally-recognised project management methodology in lreland: The view of the project manager. International Journal of Project Management, 29(5), 637-646. http://dx.doi. org/10.1016/j.jproman.2010.05.001

Moraes, R. 0., \& Kruglianskas, 1. (2012). 0 gerente de projetos de $\mathrm{Tl}$ em organizações com níveis de maturidade diferenciados. Produção, 22(4), 839-850. http://dx.doi. org/10.1590/S0103-65132012005000060

Office of Government Commerce - OGC. (1996). PRINCE2 - Projects in Controlled Environments. Londres: OGC.

Papke-Shields, K. E., Beise, C., \& Quan, J. (2010). Do project managers practice what they preach, and does it matter to project success? International Journal of Project Management, 28(7), 650-662. http://dx.doi. org/10.1016/j.ijproman.2009.11.002 
Patah, L. A., \& Carvalho, M. M. (2007). Quantifying the value of project management: The actual situation in the it market in Brazil. Ankara: EUROMA.

Pinto, S. A. 0. (2002). Gerenciamento de projetos: Análise dos fatores de risco que influenciam o sucesso de projetos de sistemas de informação (Dissertação de Mestrado). Faculdade de Economia, Administração e Contabilidade, Universidade de São Paulo, São Paulo.

Project Management Institute - PMI. (2008). A Guide to the project management body of knowledge (PMBoK Guide) (4th ed.). Project Management Institute, Four Campus Boulevard, Newtown Square.

Project Management Institute - PMl. (2009). PMl today June 2009. Project Management Institute, Four Campus Boulevard, Newtown Square.

Rabechini Junior, R., \& Carvalho, M. M. (2012). Relacionamento entre gerenciamento de risco e sucesso de projetos. Produção, 23(3), 570-581.

Rad, P. F., \& Levin, G. (2006). Metrics for project management - Formalized approaches. Management Concepts. Vienna.

Raz, T., Shenhar, A. J., \& Dvir, D. (2002). Risk management, project success, and techonological uncertainty. $R \& D$ Management, 32(2), 101-109. http://dx.doi. $\operatorname{org} / 10.1111 / 1467-9310.00243$

Repiso, L., Setchi, R., \& Salmeron, J. (2007). Modelling $I T$ projects success: Emerging methodologies reviewed. Technovation. In press.

Sanchez-Losado, J. M. (2012). Project management models: lean thought project management. DYNA, 87(2), 214221.
Silveira, G. A. (2008). Fatores contribuintes para a maturidade em gerenciamento de projetos: Um estudo em empresas brasileiras (Tese de Doutorado). Faculdade de Economia, Administração e Contabilidade, Universidade de São Paulo, São Paulo.

Söderlund, J. (2004). Building theories of project management: Past research, questions for the future. International Journal of Project Management, 22, 183191. http://dx.doi.org/10.1016/S0263-7863(03)00070-X

Standish Group International. (2009). CHAOS Summary 2009. Retrieved from http://www.standishgroup.com/.

Thamhain, H. J. (2004). Linkages of project environment to performance: Lessons for team leadership. International Journal of Project Management, 22, 533-544. http:// dx.doi.org/10.1016/j.ijproman.2004.04.005

Thomas, J., Delisle, C. L., \& Jugdev, K. (2002). Selling project management to senior executives. Project Management Institute lnc., Newtown Square.

Thomas, J., \& Mullaly, M. (2008). Researching the value of project management. PMI. Pennsylvania: Newtown Square.

White, D., \& Fortune, J. (2002). Current practice in project management - An empirical study. International Journal of Project Management, 20, 1-11. http://dx.doi. org/10.1016/S0263-7863(00)00029-6

\section{Agradecimentos}

Agradecemos aos avaliadores do trabalho, que muito contribuíram para a pesquisa com seus comentários e sugestões. Agradecemos também à CAPES e ao CNPq o suporte ao projeto de pesquisa.

\title{
Success obtained from investments in the project management methodology
}

\begin{abstract}
The objective of this work is to investigate the relationship between the adoption of project management methodologies and the project's success. The methodological approach includes a longitudinal field research conducted in 3 countries: Argentina, Brazil and Chile, during a period of 3 years, in just one organization with full access to the information. Data from 1387 projects were obtained. The results show a positive and significant influence of the variables training and number of PMPs (Project Management Profissionals) on the success of the projects and a positive influence of the project methodology used on the time results, indicating benefits to the analyzed organization.
\end{abstract}

\section{Keywords}

Project management. Performance indicators. Maturity models. 
Anexo 1. Instrumento de coleta de dados.

\begin{tabular}{|c|c|c|c|c|c|c|c|c|c|c|}
\hline$\frac{\stackrel{0}{\mathscr{2}}}{\frac{2}{2}}$ & $\stackrel{\&}{\&}$ & $\frac{\mathscr{\Xi}}{\stackrel{\Xi}{E}}$ & 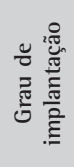 & 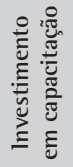 & 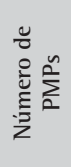 &  & 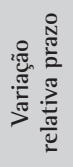 & 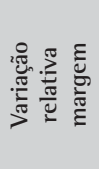 & 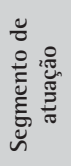 & 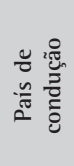 \\
\hline & & & & & & & & & & \\
\hline & & & & & & & & & & \\
\hline & & & & & & & & & & \\
\hline & & & & & & & & & & \\
\hline & & & & & & & & & & \\
\hline & & & & & & & & & & \\
\hline & & & & & & & & & & \\
\hline & & & & & & & & & & \\
\hline & & & & & & & & & & \\
\hline & & & & & & & & & & \\
\hline & & & & & & & & & & \\
\hline & & & & & & & & & & \\
\hline & & & & & & & & & & \\
\hline & & & & & & & & & & \\
\hline & & & & & & & & & & \\
\hline & & & & & & & & & & \\
\hline & & & & & & & & & & \\
\hline & & & & & & & & & & \\
\hline & & & & & & & & & & \\
\hline
\end{tabular}

Anexo 2. Informações do projeto.

\begin{tabular}{|c|c|}
\hline Tipo & Descrição \\
\hline Nome do projeto & $\begin{array}{l}\text { Referência segundo a qual o projeto é conhecido dentro da organização. Essa informação foi } \\
\text { alterada por um valor numérico sequencial por questões de privacidade das informações dos projetos } \\
\text { e dos clientes para a organização. }\end{array}$ \\
\hline Período de realização do projeto & $\begin{array}{l}\text { Medido em ano e trimestre de início de realização do projeto. A informação do trimestre de } \\
\text { realização do projeto é apresentada da seguinte forma: } \\
\text { - Q1: } 1^{\circ} \text { trimestre; periodo que compreende os meses de outubro, novembro e dezembro do } \\
\text { ano anterior ao citado na planilha, de acordo com o calendário fiscal da empresa, que se } \\
\text { inicia em outubro de um ano e termina em setembro do ano seguinte; } \\
\text { - Q2: } 2^{\circ} \text { trimestre; período que engloba os meses de janeiro, fevereiro e março; } \\
\text { - Q3: } 3^{\circ} \text { trimestre; composto pelos meses de abril, maio e junho; } \\
\text { - Q4: } 4^{\circ} \text { trimestre; compreendido pelos meses de julho, agosto e setembro. }\end{array}$ \\
\hline Grau de implementação & Grau de implementação de métodos na divisão que conduziu o projeto em valor percentual. \\
\hline $\begin{array}{l}\text { Investimento em treinamento e } \\
\text { capacitação }\end{array}$ & $\begin{array}{l}\text { Valor financeiro em reais do investimento realizado em treinamento e capacitação de gestores de } \\
\text { projetos na divisão que conduziu o projeto. }\end{array}$ \\
\hline $\begin{array}{l}\text { Número de gerentes de projetos } \\
\text { certificados como PMP }\end{array}$ & $\begin{array}{l}\text { Quantidade de profissionais certificados PMP pelo PMl na divisão, não necessariamente na condução } \\
\text { do projeto analisado em específico. }\end{array}$ \\
\hline Variação relativa do custo do projeto & $\begin{array}{l}\text { Medida através da variação relativa do aumento de orçamento, calculada pela diferença em valor } \\
\text { monetário do orçamento original previsto pelo projeto em relação ao orçamento final do projeto, } \\
\text { dividida pelo orçamento inicial. }\end{array}$ \\
\hline Variação relativa do prazo do projeto & $\begin{array}{l}\text { Medida por meio da variação relativa do aumento de prazo do projeto, calculada através da } \\
\text { diferença entre o prazo original planejado em dias e o prazo final do projeto, dividida pelo prazo } \\
\text { inicial. }\end{array}$ \\
\hline $\begin{array}{l}\text { Variação relativa da margem do } \\
\text { projeto }\end{array}$ & $\begin{array}{l}\text { Medida por meio da variação relativa da margem do projeto, calculada através da diferença entre a } \\
\text { margem final do projeto e a margem prevista originalmente, dividida pela margem inicial. }\end{array}$ \\
\hline Segmento de atuação & $\begin{array}{l}\text { Mercado onde o projeto foi aplicado. Apresentado em componentes, energia, indústria, manutenção, } \\
\text { medicina, óleo e gás, segurança, tecnologia da informação, telecomunicações e transportes. }\end{array}$ \\
\hline País de condução do projeto & País onde o projeto foi conduzido, Argentina, Brasil ou Chile. \\
\hline
\end{tabular}

